九州大学学術情報リポジトリ

Kyushu University Institutional Repository

\title{
An Architecture Framework for an Adaptive Extensible Processor
}

Noori, Hamid

Graduate school of Information Science and Electrical Engineering, Kyushu University

http://hdl. handle. net/2324/9164

出版情報 : SLRC プレゼンテーション，2007-07-25

バージョン：

権利関係 : 


\title{
An Architecture Framework for an
} Adaptive Extensible Processor

\author{
Hamid Noori
}

Graduate School of Information Science and Electrical

Engineering, Department of Informatics

Kyushu University 


\section{Outline}

- Motivations

- Goal

- An Adaptive Extensible Processor

- Extensible Processors

- General Overview of Proposed Architecture

- Generating Custom Instructions

- Proposed Reconfigurable Functional Unit

- Evaluation Results 


\section{Motivations (1/2)}

- Exploding NRE Costs
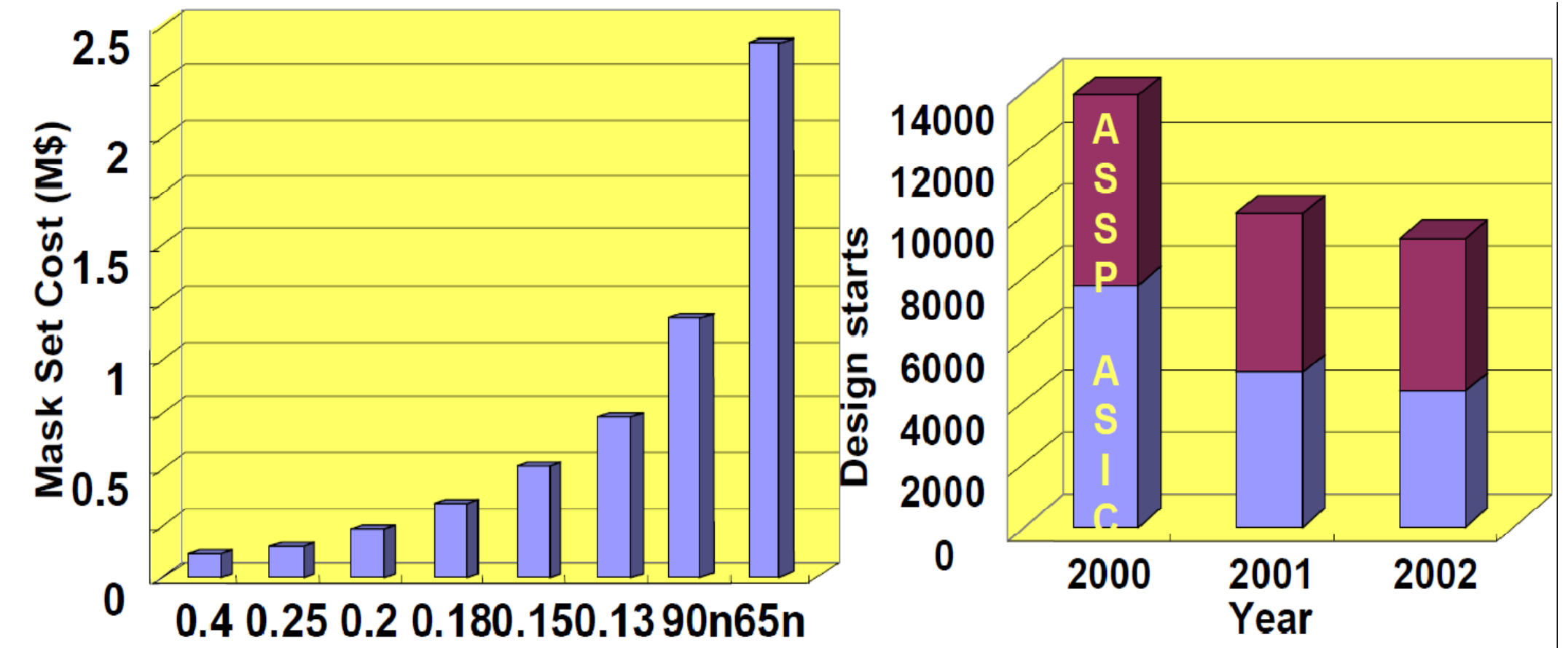

Keynote @ ASP-DAC 2007 


\section{Motivations (2/2)}

- Higher design costs due to more complex SoCs

- More complex applications $\rightarrow$ more computation and

- $\mathrm{S}$ This has led to the quest for a flexible and reusable embedded processor that still must achieve the required performance and energy efficiency levels.
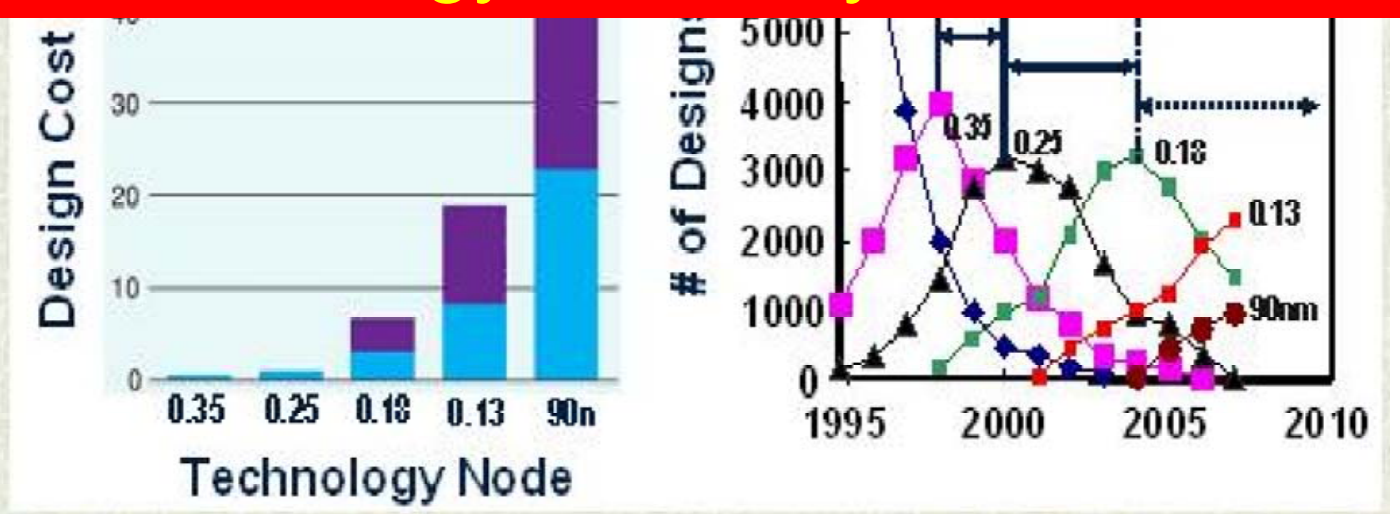

Keynote@ @ DATE 2007 


\section{Goal}

- Improving the performance and energy efficiency of embedded processors, while maintaining binary compatibility, and flexibility of embedded processors. 


\section{An Adaptive Extensible Processor}

- Introduction to Extensible Processors
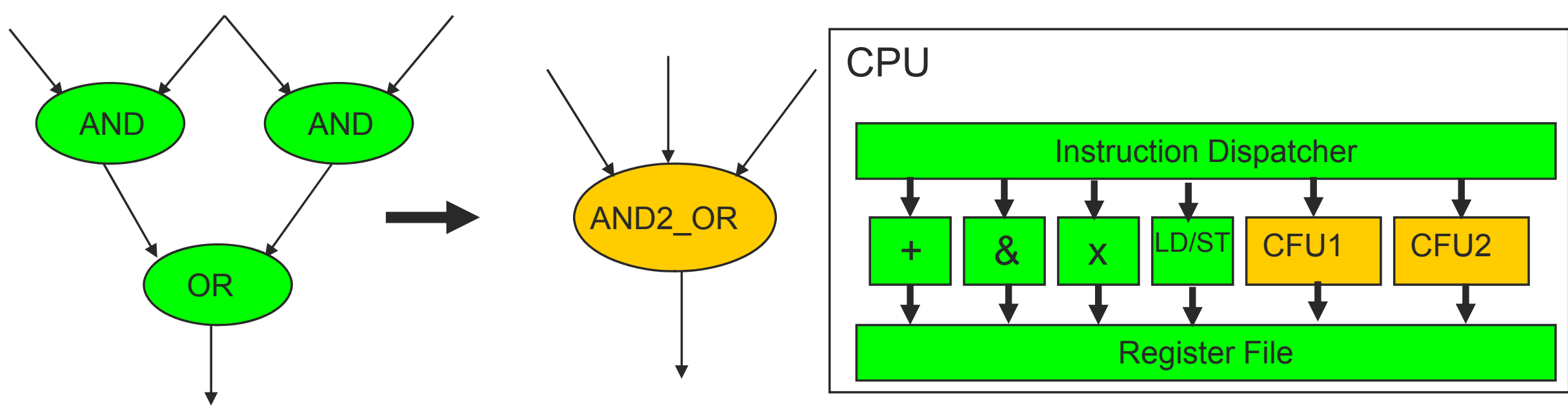

LD/ST: Load / Store

CFU: Custom Functional Unit 


\section{Custom Instructions}

- Improve performance efficiency

$\checkmark \quad$ Increasing parallelism

- Reducing the latency of critical path

- Reducing number of intermediate results written to the register file

- Reducing cache misses

- Improve energy efficiency

$\circ \quad$ Reducing accesses to

- Instruction cache

- Register file

- Decoder

- ALU

- Cache misses

- Reducing execution time (clock energy) 


\section{Proposed Approach}

- An Adaptive Extensible Processor (ADEXOR)

- Adding and generating custom instructions after fabrication

- Using a reconfigurable functional unit (RFU) instead of custom functional unit

CFU: Custom Functional Unit

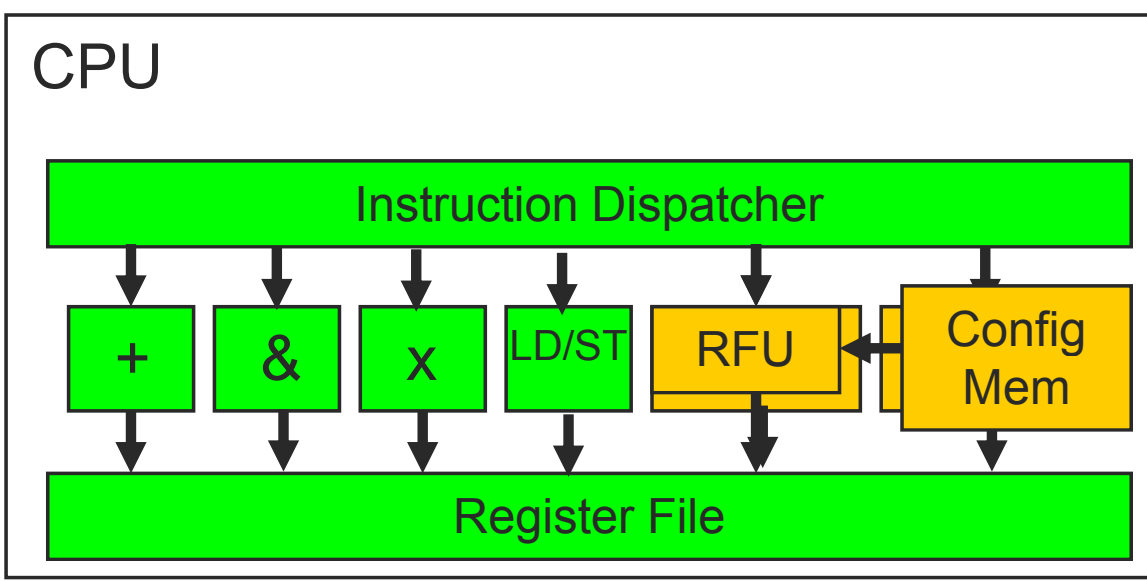




\section{General Overview of the Proposed Architecture}

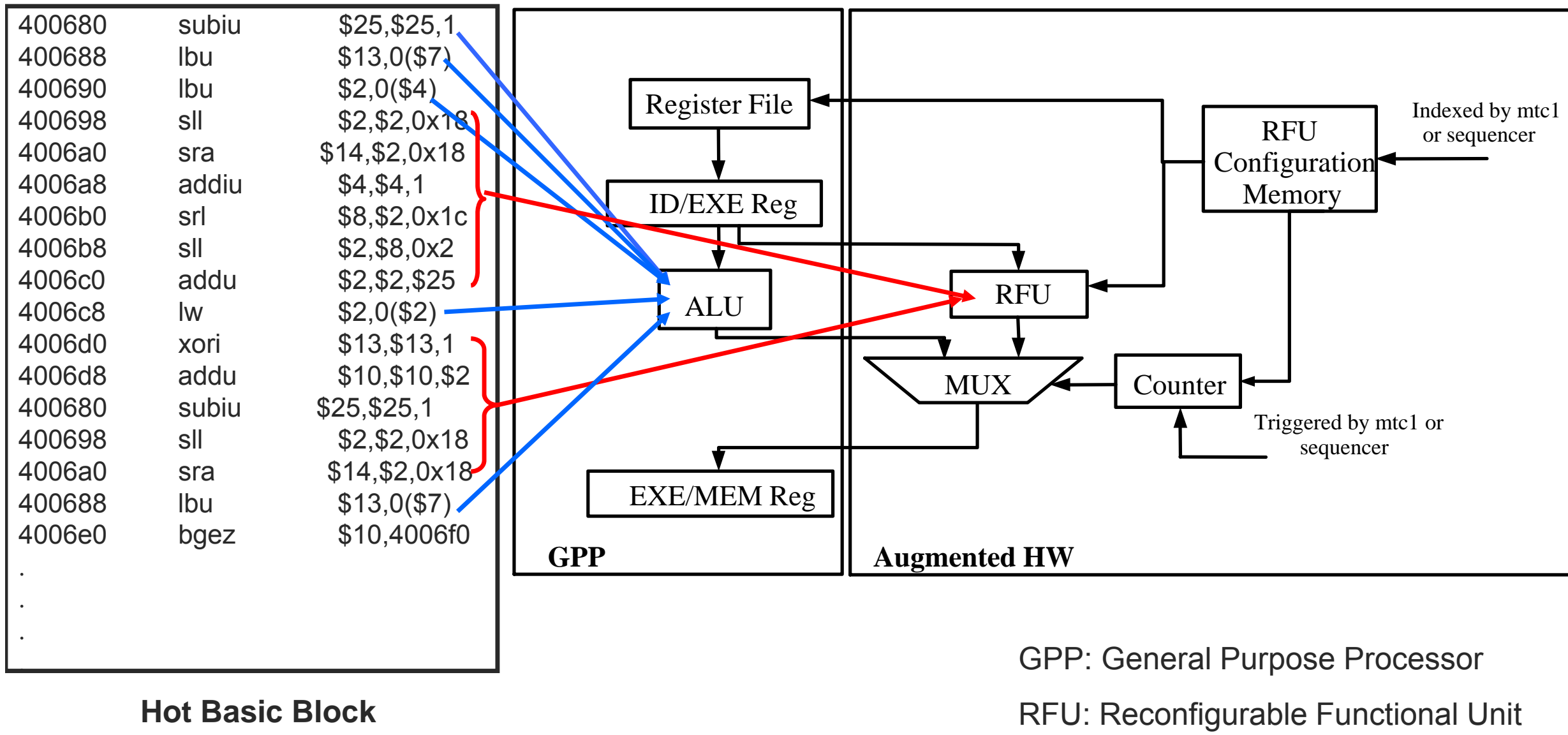

Kyushu University 


\section{Generating Custom Instructions}

- Load, divide, multiply, floating point operations are not supported

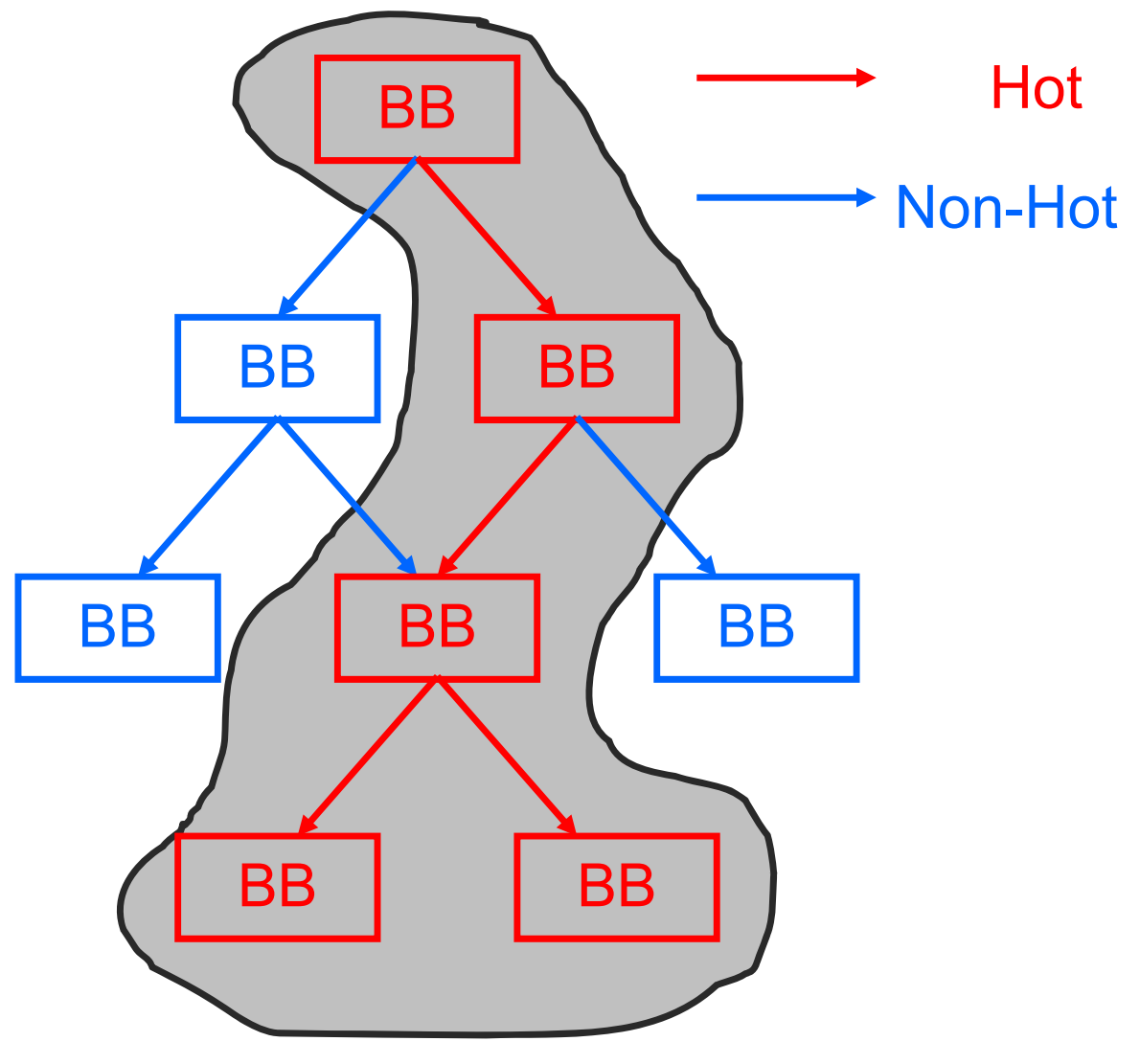

BB: Basic Block 


\section{Architecture of the RFU}

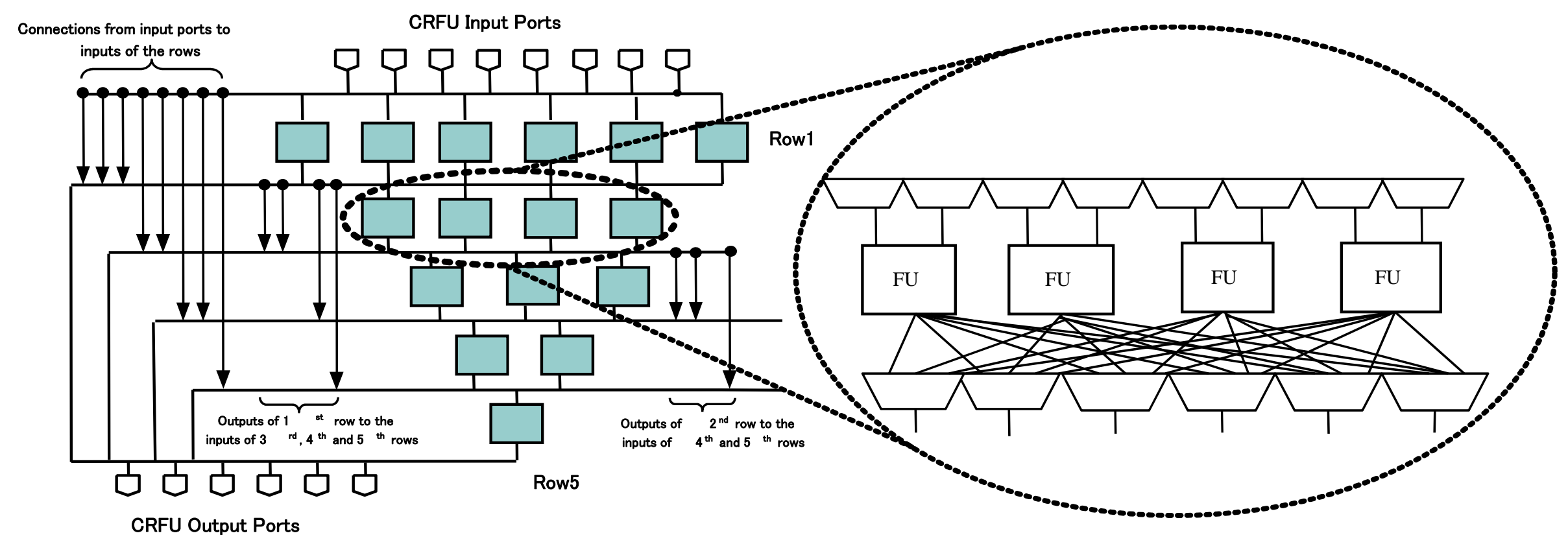

凿/// Kyushu University 


\section{Evaluation Results}

\begin{tabular}{|c|c|}
\hline Issue & 1-way \\
\hline L1- I cache & $\begin{array}{r}16 \mathrm{~K}, 2 \text { way, } 1 \text { cycle latency for hit, } \\
20 \text { cycles for miss }\end{array}$ \\
\hline L1- D cache & $\begin{array}{c}16 \mathrm{~K}, 4 \text { way, } 1 \text { cycle latency for hit, } \\
20 \text { cycles for miss }\end{array}$ \\
\hline Execution units & $\begin{array}{c}1 \text { integer unit, } 1 \text { floating point unit } \\
1 \text { divider (8 cycles), } \\
\text { multiplier (5 cycles) }\end{array}$ \\
\hline Branch predictor & bimodal \\
\hline Branch prediction table size & 256 \\
\hline Extra branch misprediction latency & 3 \\
\hline Clock frequency & $135 \mathrm{MHz}$ \\
\hline
\end{tabular}




\section{Performance Evaluation}

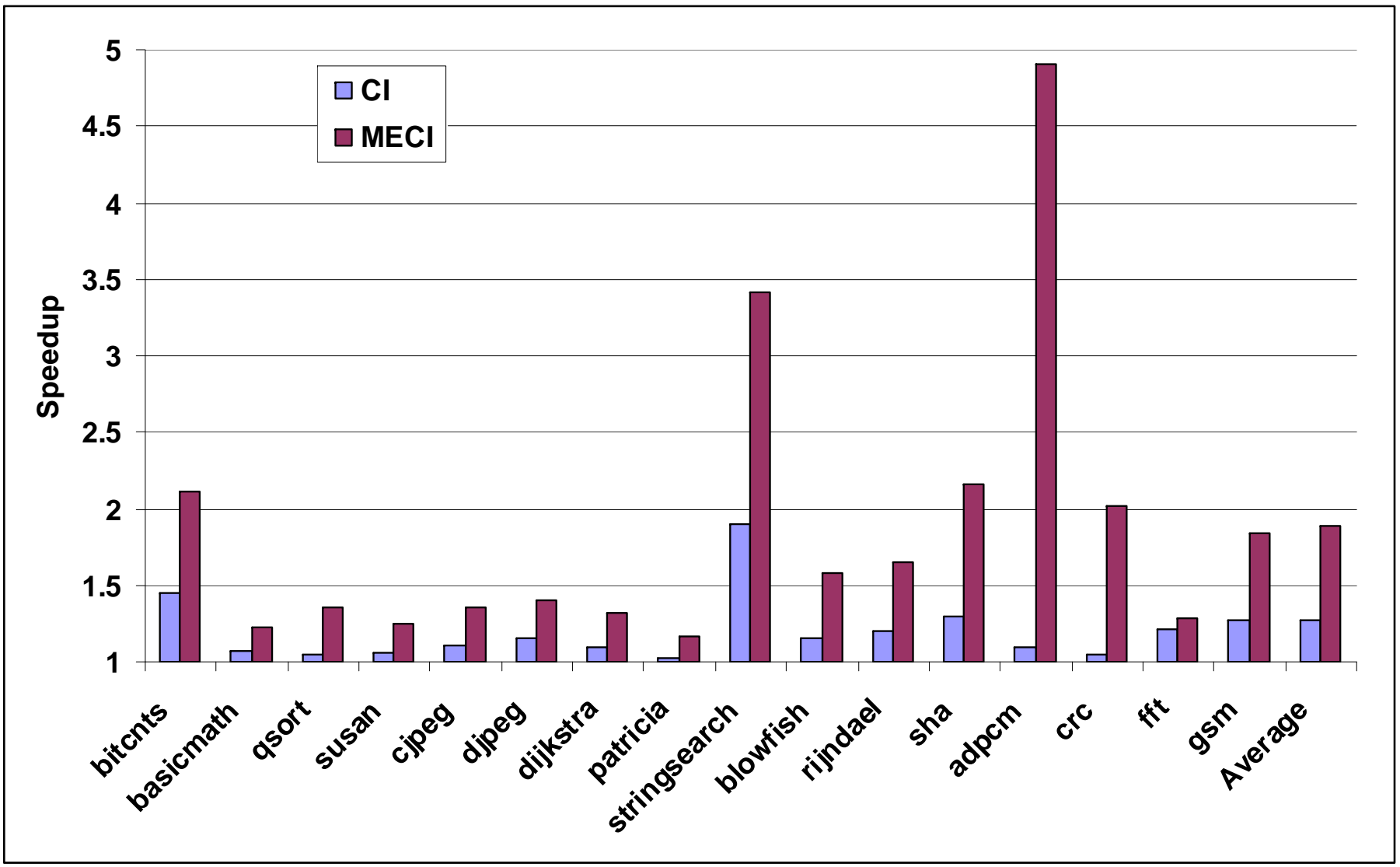




\section{Energy Evaluation}

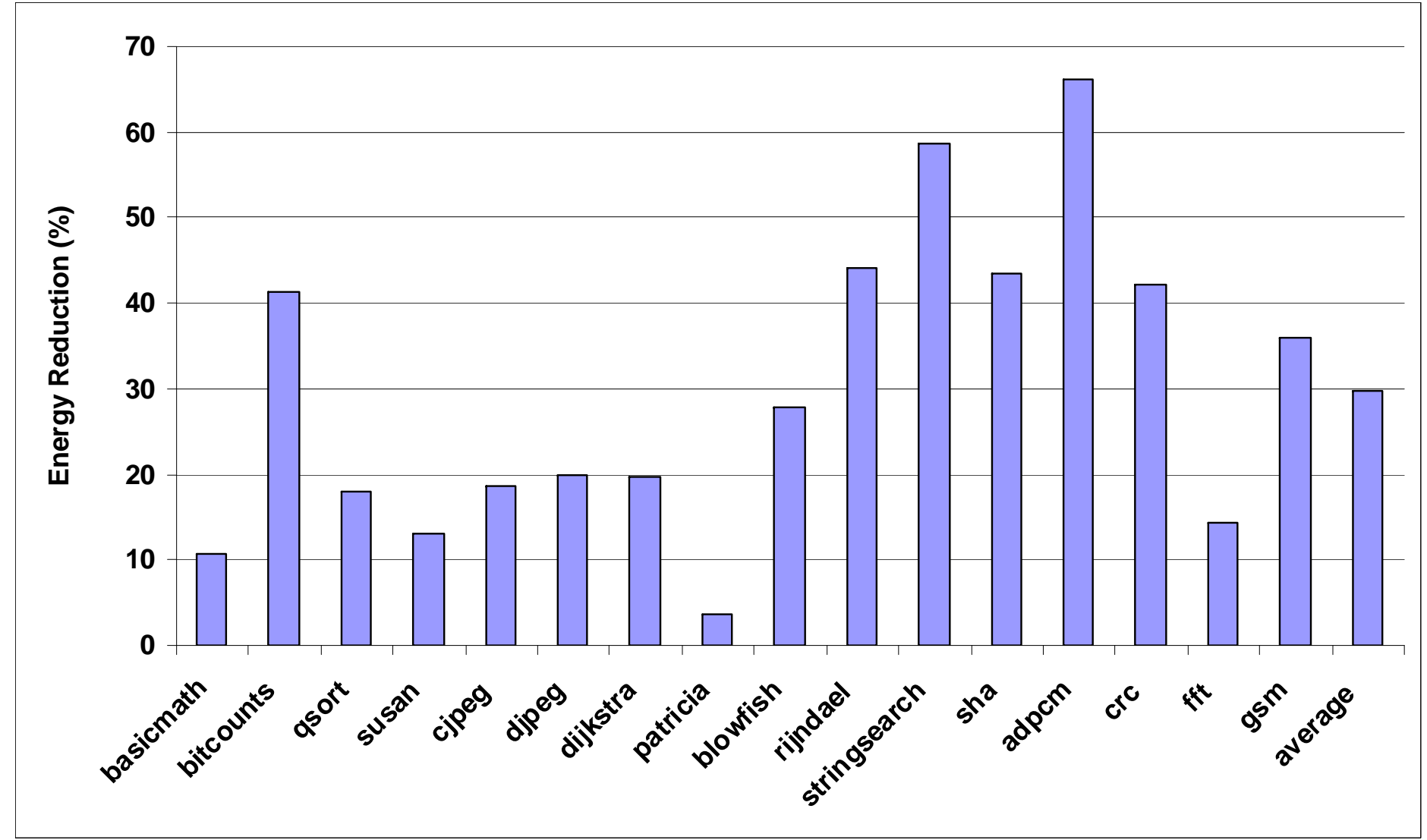




\section{Area Overhead}

- Base Processor 0.18 $\mu \mathrm{m}\left(4.5 \mathrm{~mm}^{2}\right)+$ Cache $\left(3.2 \mathrm{~mm}^{2}\right)=7.7 \mathrm{~mm}^{2}$

- Area Overhead: $37 \%$ 


\section{Conclusions}

- An architecture framework for an adaptive extensible processor

- Generating and adding custom instructions after chip-fabrication

- No new compiler, no source code modification and recompiling

- Evaluation

- Speedup: max. 4.9 and average 1.9

$\circ$ Energy saving: max. $67 \%$ and $30 \%$ in average

- Area overhead: $36 \%$ 


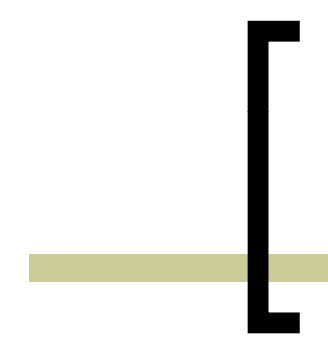

\section{Thank you for your attention}

